\title{
Transparência da água em viveiros de Macrobrachium amazonicum sob diferentes níveis de arraçoamento e despescas seletivas
}

\author{
Erlei Cassiano Keppeler \\ Universidade Federal do Acre, Centro Multidisciplinar \\ Colônia São Francisco, Gleba \\ CEP 69980-000, Campus de Cruzeiro do Sul - AC, Brasil \\ erleikeppeler@gmail.com
}

Submetido em 29/12/2007

Aceito para publicação em 23/07/2008

\section{Resumo}

Nos sistemas em aqüicultura a transparência é afetada pela água de abastecimento, ração e fertilização. Para verificar tal hipótese, foram estudados viveiros de cultivo de Macrobrachium amazonicum, no período de 19 de dezembro de 2003 a 12 de maio de 2004, por um período de aproximadamente 120 dias. O experimento foi conduzido em doze viveiros de fundo natural, com 0,01 ha e profundidade média de $1 \mathrm{~m}$. Os animais foram alimentados com ração extrusada na proporção de 6 a $9 \%$ da biomassa até a $14^{\text {a }}$ semana e, em seguida, os camarões contidos em cada três viveiros foram arraçoados com 3, 5 e 7\% da biomassa total. Outros três viveiros foram submetidos à despesca mista. A quantidade de luz penetrante foi calculada pela equação de Lambert's Law. Na análise de dados utilizou-se o teste de Kruskall-Wallis, complementado com LSD, observando-se diferença significativa entre os tratamentos 3 e $5 \%(\mathrm{p}<0,05)$. Para a despesca seletiva, utilizando o teste de Mann-Whitney, não se observou diferença estatística entre os tratamentos. O percentual de absorção da luz foi geralmente maior que $90 \%$. Quanto aos estratos, considerados como superfície e fundo para temperatura, $\mathrm{pH}$ e oxigênio dissolvido, não foram observadas diferenças estatísticas significativas nestes estratos entre as duas zonas $(\mathrm{p}>0,05)$.

Unitermos: absorção da luz, produtividade, viveiros de Macrobrachium amazonicum

\section{Abstract}

Water transparency of Macrobrachium amazonicum ponds under different feeding regimens and selective harvests. In aquaculture systems, water transparency is affected by water supply, feeding rate and fertilization. A study was conducted from December 19, 2003 to May 12, 2004 for a period of approximately 120 days. Twelve 0.01 ha earthen ponds were used with $1 \mathrm{~m}$ deep water. Prawns were fed commercial diet at a rate of 7 to $9 \%$ of biomass until the $14^{\text {th }}$ week. Then, three groups of three ponds were fed with $3 \%, 5 \%$ and $7 \%$ of prawn biomass. The remaining three other ponds were subjected to combined harvest. The amount of penetrating light was calculated according to Lambert's Law, described as $I_{z}=I_{0} e^{-k z}$. Evaluation of the data using Kruskall-Wallis, complemented by LSD test, showed a significant difference between treatments of 3 and $5 \%(\mathrm{p}<0.05)$. The Mann-Whitney Test was also used for selective harvest and no difference was observed between treatments. The percentage of light absorbed was generally greater than $90 \%$. With regard to the water layers, the surface and bottom were examined for temperature, $\mathrm{pH}$ and dissolved oxygen, but no statistically significant difference was found between the two zones ( $\mathrm{p}>0.05)$.

Key words: light absorbed, productivity, Macrobrachium amazonicum ponds 


\section{Introdução}

A visibilidade do disco de Secchi é basicamente uma função da reflexão da luz na superfície do disco, que é afetada pelas características de absorção da água e da matéria orgânica particulada e dissolvida (Wetzel e Likens, 1991). Nos sistemas em aqüicultura, a transparência da água é influenciada pelo material proveniente da água de abastecimento, pela alimentação artificial constituída pela ração balanceada, e ainda a fertilização química (Zimmermann, 1998).

Segundo Zimmermann (1998), na fase de crescimento em sistemas de cultivo semi-intensivos, a produção primária de um viveiro desempenha papel de importância na manutenção da qualidade de água. Por isso, as populações de fitoplâncton devem ser mantidas em níveis adequados (Valenti, 1985; Zimmermann, 1998).

O fitoplâncton apresenta papel fundamental no metabolismo das comunidades aquáticas, pois é responsável pela produção de matéria orgânica e oxigênio (Wetzel, 1981; Margalef, 1983). A concentração deste gás e o $\mathrm{pH}$ resultam de um balanço entre os processos de fotossíntese e respiração. Esses parâmetros são extremamente influenciados pelas algas e apresentam uma estratificação espacial e temporal nos viveiros de carcinicultura (Valenti, 1998). A estratificação espacial define um estrato mais superficial e um estrato situado próximo ao fundo (Piedrahita et al., 1984).

Estas zonas podem também apresentar diferenças de temperatura entre a superfície e o fundo (Zimmermann, 1998). Conforme Boyd (1979), a ausência ou a presença da estratificação térmica em viveiros depende da mistura da água pelo vento e do fluxo.

O objetivo do presente trabalho foi analisar a transparência e a absorção de luz de 12 viveiros de carcinicultura. Além disso, verificaram-se outras características hidrológicas como, temperatura, $\mathrm{pH}$ e oxigênio dissolvido na superfície e fundo. Adicionalmente, determinaram-se os efeitos de diferentes taxas de ração (3, 5 e 7\%) e despesca seletiva sobre a transparência.

\section{Material e Métodos}

O experimento foi conduzido no Setor de Carcinicultura (Figura 1), no período de dezembro/2003 a maio/2004. Foram utilizados doze viveiros de 0,01 ha \pm 0,01 , escavados com fundo de terra e paredes inclinadas em ângulos de $45^{\circ}$ e cobertas por vegetação rasteira para protegê-las da erosão. Estes foram construídos sobre latossedimento vermelho-escuro com textura argilosa.

Para aumentar a produtividade primária, e assim incrementar a biomassa final, os viveiros foram submetidos à calagem, com calcário dolomítico, distribuindo-se $500 \mathrm{~kg} / \mathrm{ha}$, e fertilização orgânica, distribuindo-se ao longo do viveiro esterco de boi bem curtido, numa quantidade de $2.000 \mathrm{Kg} / \mathrm{ha}$. Posteriormente, realizou-se o povoamento com densidade de 20 ind. $\mathrm{m}^{-2}$, onde foram estocadas pós-larvas preliminarmente com diferentes tamanhos de $M$. amazonicum, na última semana de dezembro. Ao longo do cultivo, os viveiros receberam adubação química para incentivar o crescimento de organismos bentônicos (larvas de insetos, anelídeos, etc.) que servem de alimento natural para o camarão. Esta adubação foi realizada com uréia + sulfato de amônia, NPK e uréia + superfosfato simples. As quantidades de $\mathrm{N}$ e P aplicadas semanalmente são apresentadas na Tabela 1 .

Telas na saída do cano de abastecimento dos viveiros foram cobertas com redes de nylon com malha de $300 \mu \mathrm{m}$ para prevenir peixes adultos, larvas e ovos. Pós-larvas de aproximadamente 50 dias de idade foram transportadas de berçários bifásicos para viveiros de crescimento final. Mensalmente foi coletada uma amostra de 3-5\% dos camarões estocados em cada viveiro. Os espécimes foram medidos em ictiômetro, com precisão de $0,1 \mathrm{~mm}$, pesados em Balança Marte A 500 , com precisão de $0,01 \mathrm{~g}$, para cálculo da ração.

Os camarões foram alimentados diariamente com ração comercial peletizada, com teor protéico de 38\%, na taxa de 7 a 9\% da biomassa contida em cada viveiro, conforme a fase de desenvolvimento, até o penúltimo mês. A ração foi dividida em duas porções iguais e distribuída geralmente às 8 e às 16 h. 


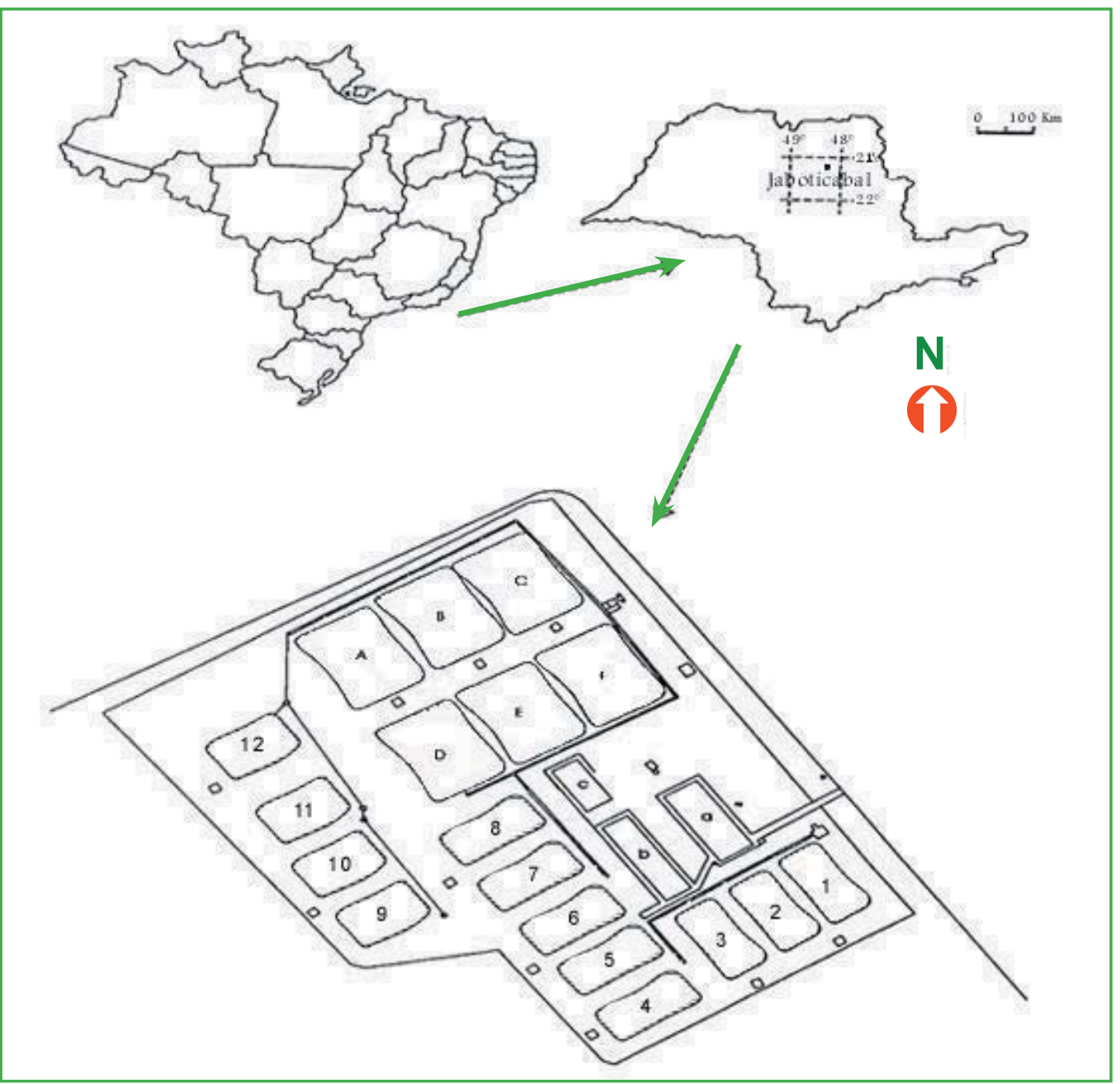

FIGURA 1: Localização da área de estudo (Legenda: 1 a 12 Viveiros de crescimento final do M. amazonicum; A a F - Viveiros usados para reprodução de M. amazonicum, M. rosenbergii e esporadicamente usado como berçário secundário; A - Laboratório de larvicultura e qualidade de água; B - Berçário; C - Laboratório de Biometria (Keppeler e Valenti, 2006).

TABELA 1: Quantidade de nitrogênio $(\mathrm{N})$ e fósforo $(\mathrm{P})$ das fertilizações semanais.

\begin{tabular}{c|c|c}
\hline Semanas & $\mathbf{N}\left(\mathbf{k g} \cdot \mathbf{h a}^{-1}\right)$ & $\mathbf{P}\left(\mathbf{k g} \cdot \mathbf{h a}^{-\mathbf{1}}\right)$ \\
\hline 3 & 8,8 & - \\
\hline 4 & 8,8 & - \\
\hline 5 & 3,12 & 0,68 \\
\hline 6 & 3,12 & 0,68 \\
\hline 9 & 3,12 & 0,68 \\
\hline 11 & 1,98 & 4,2 \\
\hline 12 & 1,98 & 4,2 \\
\hline 14 & 1,98 & 4,2 \\
\hline
\end{tabular}

A biomassa foi estimada por meio de biometria mensal e corrigida nos dois primeiros meses, quinzenalmente no terceiro mês, e semanalmente no último mês de engorda.

O delineamento experimental para o estudo dos diferentes níveis de arraçoamento foi totalmente casualizado com três tratamentos (taxas de alimentação) e três repetições. Cada três viveiros, sorteados ao acaso, foram arraçoados no último mês de cultivo com 3, 5 e $7 \%$ da biomassa de camarões estimada por biometria.

Concomitantemente, três viveiros foram submetidos ao sistema de despesca mista. Quando parte da população atingiu o tamanho comercial, em meados de março, foram iniciadas as despescas seletivas. Estas consistiram 
em despescas quinzenais, com redes de arrasto com malha 15 e $18 \mathrm{~mm}$ para a retirada de animais maiores dos viveiros (Valenti e New, 2000). Ambas com malha inferior com chumbo por metro linear. Este processo de despesca visa acelerar o crescimento dos camarões menores, que é inibido na presença de animais grandes nos viveiros.

As despescas seletivas foram realizadas nas semanas 12,14 e 16; 1 a 3 dias antes das coletas de água. Em meados de maio, decorridos 145 dias do povoamento, foi realizada a despesca total, em todos os viveiros. Após as despescas, os animais foram contados e pesados. Depois, foi determinada a produção para cada viveiro. Nos viveiros submetidos à despesca mista, esta representou a soma de todas as despescas.

A transparência da água dos viveiros foi determinada às $11 \mathrm{~h}$. Para isso, utilizou-se disco de Secchi de $20 \mathrm{~cm}$ de diâmetro, dividido em quadrantes alternados pretos e brancos. Foi calculado o coeficiente de extinção da luz através da relação $K=1,7 / Z$, onde $Z$ é a profundidade do disco de Secchi e 1,7 constante (Poole e Atkins, 1929), derivados da fórmula descrita por Margalef (1983):

$$
\mathrm{K}=0,03+0,015 \text { (clor.) }+\sum 34 \mathrm{Vi} / \mathrm{di}=1,7 / \mathrm{Z}
$$

Onde:

$\mathrm{K}$ é o coeficiente de extinção

0,03 é a extinção da água a $540 \mathrm{~nm}$

0,015 é a extinção da clorofila mg. $\mathrm{m}^{-3}$

$\sum 34 \mathrm{Vi} /$ di é a extinção devido às partículas em suspensão

Dados quantitativos também foram determinados, como a percentagem de absorção da luz (Wetzel, 1981), baseada na equação:

$$
\text { \% Absorção }=100\left(I_{o}-I_{z}\right) / I_{o}
$$

Onde:

$\mathrm{I}_{0}=$ penetração da radiação na superfície

$\mathrm{I}=$ radiação à profundidade $\mathrm{z}$

Absorção da percentagem foi originalmente usada para estudar a penetração da luz monocromática, mas o conceito pode ser estendido para a radiação total.
A quantidade de luz penetrante para qualquer profundidade $\mathrm{Z}$ pode ser calculada pela equação de Lambert's Law (Hutchinson, 1957):

$$
\mathrm{I}_{\mathrm{z}}=\mathrm{I}_{\mathrm{o}} \mathrm{e}^{-\mathrm{kz}} \text { ou } \mathrm{LnI}_{\mathrm{o}}-\mathrm{LnI}_{\mathrm{z}}=\mathrm{K}_{\mathrm{z}}
$$

Onde:

$\mathrm{e}=$ base dos logarítmos naturais

A produtividade integrada também foi determinada, utilizando-se a fórmula $\mathrm{K} \times \mathrm{P}_{\mathrm{m}} \times \mathrm{D}_{\text {Sechii }}$, calculando-se o Carbono, segundo Wetzel (1981).

A alcalinidade foi determinada pela metodologia descrita por Boyd (1982), e o carbono calculado segundo Boyd e Tucker (1992). A temperatura e o oxigênio dissolvido foram mensurados com oxímetro, o pH com peagômetro. $\mathrm{O}$ tempo de residência da água foi calculado conforme Ribeiro (2001).

\section{Análise estatística}

O tratamento estatístico dos dados foi feito a partir de análise descritiva através dos cálculos da média aritmética e da mediana como medidas de tendência central, assim como intervalos de confiança. Calculouse o coeficiente de correlação Linear de Pearson (r) da transparência com a quantidade média de ração fornecida semanalmente (Zar, 1984).

Com a finalidade de estabelecer o nível de significância da temperatura, oxigênio e $\mathrm{pH}$ nas zonas superfície e fundo foram realizados testes. Preliminarmente, utilizou-se o teste Shapiro Wilk. Este mostrou que as variáveis obtidas não seguiram uma distribuição normal. Foi realizado o teste de MannWhitney (Zar, 1984).

Para a comparação da transparência nos diferentes tratamentos, usou-se o delineamento experimental inteiramente casualizado, com três tratamentos e 3 repetições. Os dados das três réplicas de cada tratamento foram também submetidos ao teste Shapiro Wilk. Como os dados não apresentaram normalidade e homogeneidade efetuou-se os Testes de Mann-Whitney e Kruskall Wallis. Este foi complementado pelo Teste LSD (Diferença Quadrada Mínima), com diferente número de repetições, para comparação dos tratamentos. As análises foram realizadas utilizando-se os softwares Statistica da 
Statsoft (1996) versão 6.0 e SAS versão 8.2 (2001), com nível de significância de 0,05 .

\section{Resultados}

As Figuras 2A e 2B apresentam a transparência da água e o coeficiente de extinção da luz. A média geral da transparência foi 0,53 . O valor mínimo foi $0,17 \mathrm{~m}$ e o valor máximo 1,0m. Quanto ao coeficiente de extinção, o valor mínimo foi 1,70 e o valor máximo 9,71.

A Figura 3 mostra a ração adicionada nos viveiros. A média geral foi $298,7 \mathrm{~kg} \cdot \mathrm{ha}^{-1} \cdot \mathrm{dia}^{-1}$. A soma da ração mínima foi $113,20 \mathrm{~kg} \cdot \mathrm{ha}^{-1} \cdot \mathrm{dia}^{-1} \mathrm{e}$ a máxima foi $744,81 \mathrm{~kg}$. $\mathrm{ha}^{-1} \cdot \mathrm{dia}^{-1}$. A Figura 4 apresenta as quantidades de nitrogênio e fósforo adicionada nos viveiros.

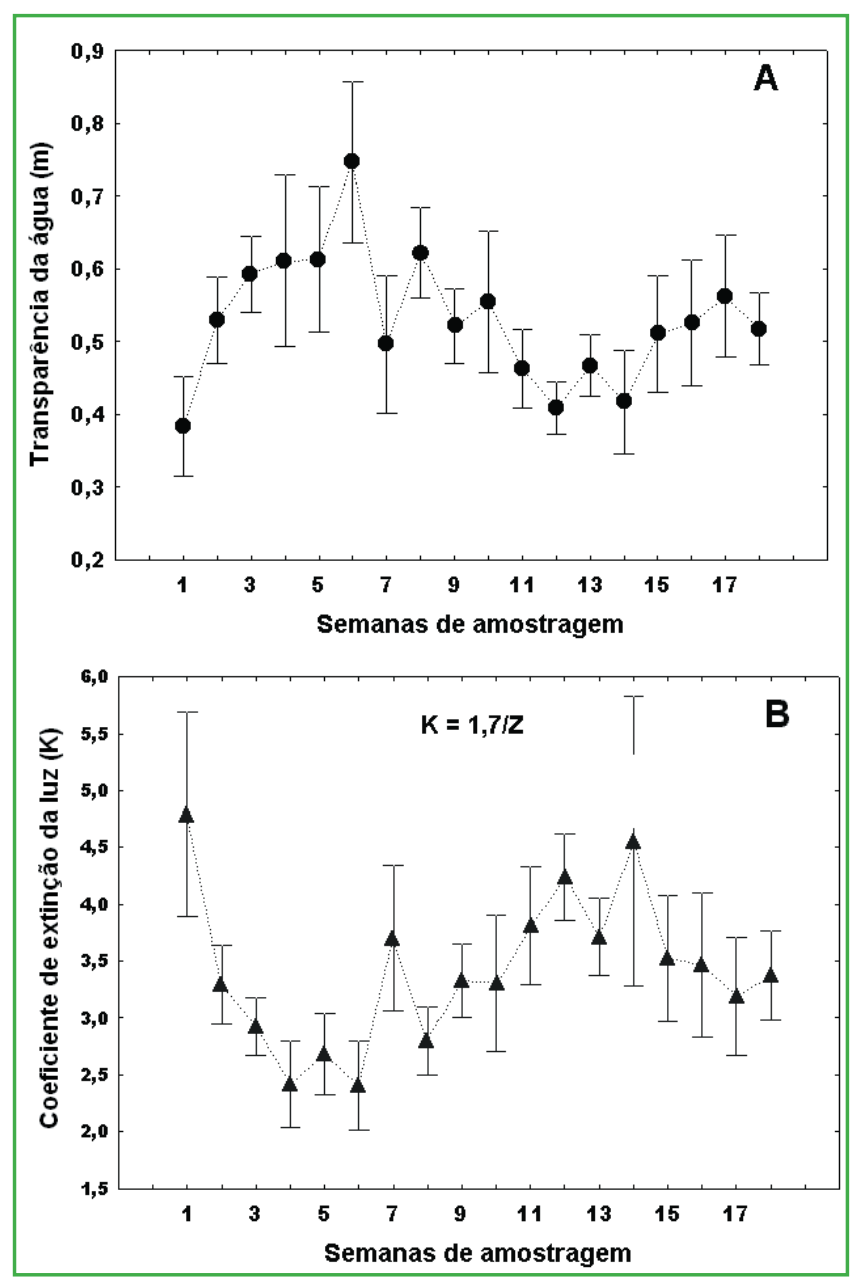

FIGURA 2: A - Transparência da água nos viveiros de $M$. amazonicum (média $\pm 0,95$ de $\mathrm{IC}=$ intervalo de confiança); B - Coeficiente de extinção da luz (K) nos viveiros de $M$. amazonicum (média $\pm 0,95$ de $\mathrm{IC}=$ intervalo de confiança).

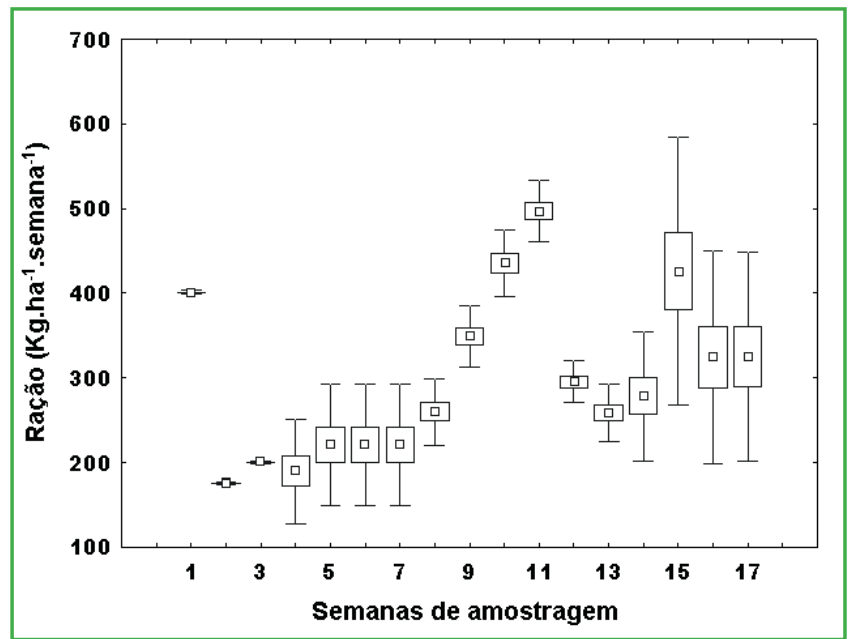

FIGURA 3: Soma da ração em função do tempo em viveiros de $M$. amazonicum.

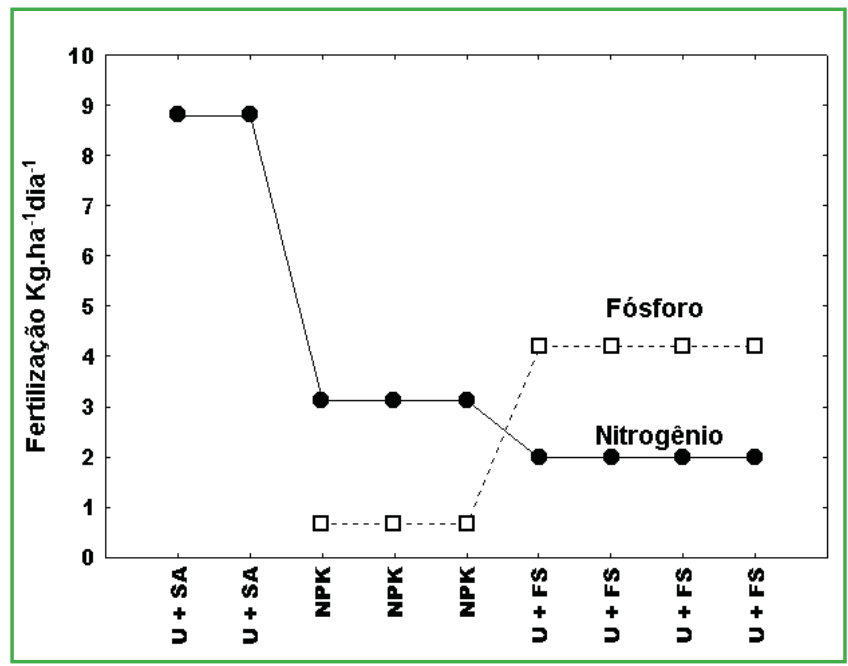

FIGURA 4: Nitrogênio e Fósforo proveniente das fertilizações químicas ( $\mathrm{U}=$ uréia; $\mathrm{SA}=$ Sulfato de amônia NPK = Nitrogênio, Fósforo e potássio (12:6:12), FS = Fosfato simples), nos viveiros de M. amazonicum.

Observaram-se correlações negativas entre a ração acumulada e a transparência $(\mathrm{p}<0,05)$ que variaram de $\mathrm{r}$ $=-0,52$ a $-0,71$. Na Figura 5 , é apresentada a absorção da luz, expressa em porcentagem, durante todo o cultivo. $\mathrm{O}$ valor mínimo foi $81,73 \%$ e o máximo $99,99 \%$. O valor médio observado foi $95,17 \%$.

A Figura 6 mostra a produtividade integrada em termos de carbono produzido por metro quadrado. $\mathrm{O}$ valor médio observado foi $1.741,59 \mathrm{mg}$. C. $^{-2}$. Verificouse que a maior produção ocorreu na última semana de amostragem alcançando $27.290 \mathrm{mg}$. C.m $\mathrm{m}^{-2}$. 


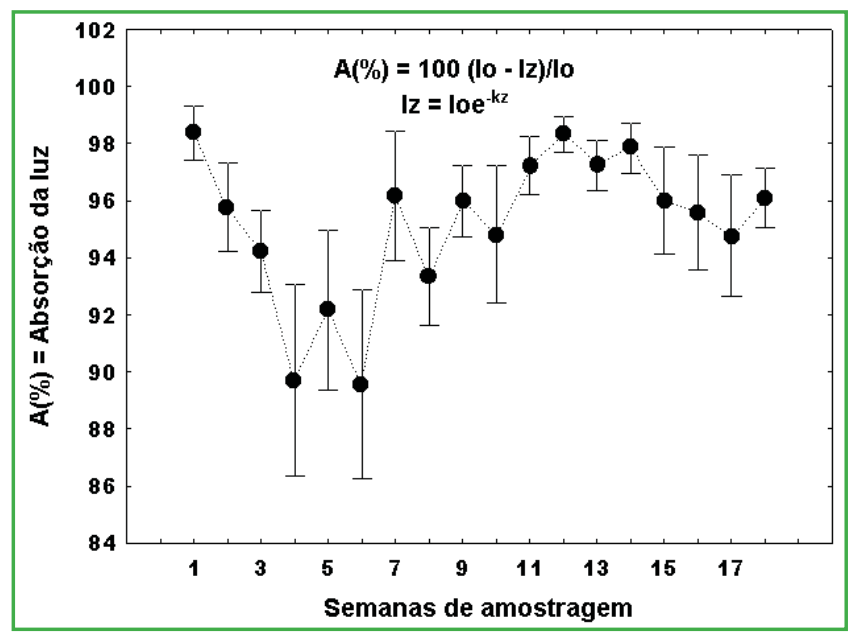

FIGURA 5: Absorção da luz (\%) em viveiros de M. amazonicum $( \pm \mathrm{IC}=$ intervalo de confiança).

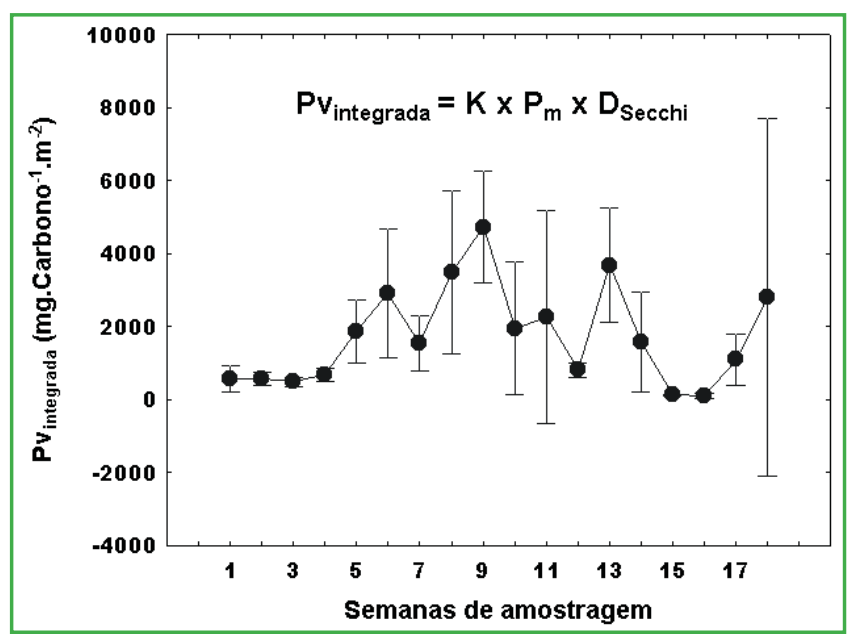

FIGURA 6: Produtividade integrada nos viveiros de M. amazonicum (média $\pm 0,95$ de intervalo de confiança).

A partir do penúltimo mês, o teste de Kruskall Wallis revelou diferença significativa para a transparência ( $\mathrm{p}$ $<0,05)$ entre os tratamentos 3 e $5 \%$ e 3 e $7 \%$. Quanto aos tratamentos de despesca seletiva e mista, o teste de Mann Whitney não revelou diferença significativa para os mesmos. Também os tratamentos e a transparência mostraram correlação significativa negativa $(r=-0,31$ e p $<0,05)$.

O tempo de residência foi em média 7,47 $\pm 22,0$ dias. Em viveiros de aqüicultura, o tempo de residência recebe a sua maior influência da taxa de renovação de água do viveiro, tomando-se por base, normalmente, a concentração de amônia, proveniente dos dejetos dos animais e da ração (Souza et al., 2001).
Quanto à alcalinidade, os valores médios estão mostrados na Tabela 2, nos viveiros no período da manhã e tarde.

TABELA 2: Valores médios da alcalinidade durante o cultivo de M. amazonicum. Dados são reportados como média e desvio-padrão.

\begin{tabular}{l|c|c}
\multirow{2}{*}{\multicolumn{1}{c|}{ Variáveis }} & \multicolumn{2}{|c}{ Viveiros } \\
\cline { 2 - 3 } & $\mathrm{M}$ & $\mathrm{T}$ \\
\hline Alcalinidade & 48,02 & 47,71 \\
$\left(\mathrm{mg} \mathrm{CaCO}_{3} \mathrm{~L}^{-1}\right)$ & $\pm 1,76$ & $\pm 1,66$ \\
\hline
\end{tabular}

$\mathrm{M}=$ Manhã e $\mathrm{T}=$ tarde

Os dados da temperatura da água, mostrados na Figura $7 \mathrm{a}$, apresentaram variações, as quais são conseqüências tanto do regime climático normal quanto das variações diurnas. Assim, observaram-se águas mais frias durante a manhã. A temperatura da água mantevese semelhante na superfície e fundo, pois não houve diferença significativa $(p>0,05)$.

Os viveiros se apresentaram como sistemas bem oxigenados, cujo teor de oxigênio dissolvido (Figura 7b) acompanhou a similaridade entre os estratos avaliados ( $\mathrm{p}$ $>0,05)$. Isso ocorreu possivelmente por serem sistemas rasos de $1 \mathrm{~m}$, com constante circulação da água, e tempo de residência relativamente curto.

$\mathrm{Na}$ Figura $7 \mathrm{c}$ são apresentados os valores medianos do potencial hidrogênio iônico $(\mathrm{pH})$, nos viveiros (superfície e fundo), obtidos ao longo do período experimental. Também não houve diferença significativa entre as profundidades testadas $(p>0,05)$. Como também o pH depende da decomposição que ocorre nos processos biológicos, o baixo tempo de residência permitiu a eliminação dos metabólicos, mantendo-o uniforme ao longo do tempo.

As Tabelas 3 e 4 apresentam a produtividade dos camarões no final do cultivo. Observamos que as maiores produtividades foram nos tratamentos de $7 \% \mathrm{e}$ despesca mista. 

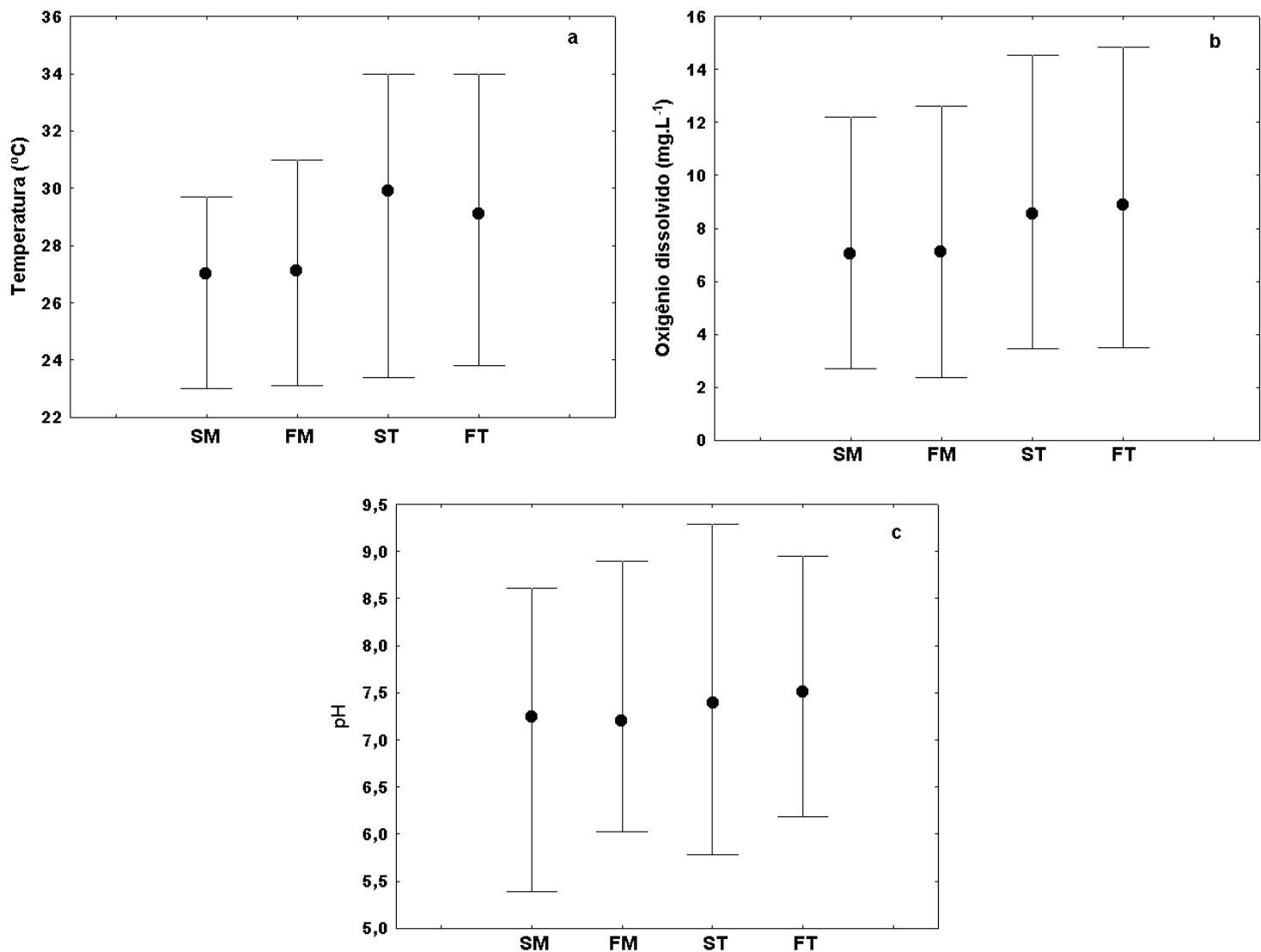

FIGURA 7: Valores medianos de temperatura (a), oxigênio dissolvido (b) e pH (c).

TABELA3: Dimensão, número de indivíduos estocados de $M$. amazonicum, produção e tratamento dos tipos de arraçoamento aplicado para cada viveiro.

\begin{tabular}{c|c|c|c|c}
\hline Viveiros & $\begin{array}{c}\text { Dimensão do } \\
\text { viveiro } \mathbf{( m}^{\mathbf{2}} \mathbf{\text { N }}\end{array}$ & $\begin{array}{c}\text { estocados } \\
\text { estuos }\end{array}$ & $\begin{array}{c}\text { Produção } \\
\left.\mathbf{( k g . h a}^{-1}\right)\end{array}$ & Tratamentos \\
\hline 2 & 89 & 1780 & 793,05 & $7 \%$ \\
\hline 3 & 92 & 1840 & 637,03 & $5 \%$ \\
\hline 4 & 100 & 2000 & 642,53 & $5 \%$ \\
\hline 5 & 101 & 2020 & 729,13 & $7 \%$ \\
\hline 6 & 102 & 2040 & 612,6 & $3 \%$ \\
\hline 7 & 106 & 2120 & 695,53 & $7 \%$ \\
\hline 9 & 124 & 2480 & 597,11 & $3 \%$ \\
\hline 10 & 115 & 2300 & 615,76 & $3 \%$ \\
\hline 12 & 98 & 1960 & 630,29 & $5 \%$ \\
\hline
\end{tabular}

TABELA 4: Dimensão, número de indivíduos estocados de M. amazonicum, produção e tratamento das despescas aplicadas para cada viveiro.

\begin{tabular}{c|c|c|c|c}
\hline Viveiros & $\begin{array}{c}\text { Dimensão } \\
\text { do viveiro } \\
\left(\mathbf{m}^{\mathbf{2}}\right)\end{array}$ & $\begin{array}{c}\mathbf{N}^{\mathbf{0}} \text { indivíduos } \\
\text { estocados }\end{array}$ & $\begin{array}{c}\text { Produção } \\
\mathbf{( k g . h a}^{-1} \mathbf{)}\end{array}$ & Tratamentos \\
\hline 1 & 89 & 1780 & 717,93 & $\begin{array}{c}\text { Despesca } \\
\text { mista }\end{array}$ \\
\hline 3 & 92 & 1840 & 637,03 & $\begin{array}{c}\text { Despesca } \\
\text { mista }\end{array}$ \\
\hline 4 & 100 & 2000 & 642,53 & $\begin{array}{c}\text { Despesca } \\
\text { total }\end{array}$ \\
\hline 8 & 100 & 2000 & 666,27 & $\begin{array}{c}\text { Despesca } \\
\text { total }\end{array}$ \\
\hline 11 & 120 & 2400 & 500,70 & $\begin{array}{c}\text { Despesca } \\
\text { mista }\end{array}$ \\
\hline 12 & 98 & 1960 & 630,29 & $\begin{array}{c}\text { Despesca } \\
\text { total }\end{array}$ \\
\hline
\end{tabular}




\section{Discussão}

Os valores relativamente baixos de transparência no final do cultivo ora detectados são indícios da baixa atenuação da radiação (Wetzel e Likens, 1991) e, conseguintemente, devido à presença de muitos compostos orgânicos dissolvidos na água (proveniente da ração), bem como da baixa penetração da luz nos viveiros de $M$. amazonicum. Dessa forma, houve no final do cultivo um decréscimo da transparência com o aumento da ração, decorrente de sua acumulação. A atenuação ocorre dispersando o comprimento de onda eletromagnética ou absorvendo-o, estando relacionada com a quantidade de material inorgânico e orgânico em suspensão na coluna d'água. Portanto, quanto menor a transparência da água implica em maior atenuação (Esteves, 1998).

A partir dos valores da transparência de Secchi, foi possível notar a presença de luz até a metade da profundidade dos viveiros durante todo o período estudado. A transparência, assim como o coeficiente de extinção foram baixos e suas variações foram decorrentes das adubações químicas realizadas, bem da quantidade de ração adicionada nos viveiros.

Os valores de transparência encontrados sugerem possível presença de plâncton, pois estiveram entre $25-60 \mathrm{~cm}$, faixa ideal para desenvolvimento de alta densidade de algas (Valenti, 1998). Possivelmente, a fertilização contribuiu para a manutenção dos valores nesta faixa. Estes valores também foram indicados por Zimmermmann (1998), Boyd e Zimmermann (2000) e Valenti e New (2002).

Além do alimento natural Delincé (1992), também salienta mais duas categorias, provenientes das fertilizações químicas e ração. Nos viveiros foram adicionados vários $\mathrm{kg}$ de Nitrogênio e Fósforo, para incremento da produção de alimento natural, e que pode ter regulado a transparência. O aumento da produtividade natural tem sido conseguido pela adição de fertilizantes químicos, para redução das necessidades de ração (Correia et al., 2003). Alimentos naturais são freqüentemente componentes da dieta para camarões de água doce (Kubitza, 2003), exceto quando há peletes de alimentos formulados (Weidenbach, 1980).
No início do cultivo a transparência foi baixa. Além disso, infere-se que existiu material em suspensão na coluna d'água, tornando-as menos transparentes, pois a água de abastecimento e os viveiros podem ter sido influenciados pelo efeito das chuvas, que ocorreram em quantidade maior nos meses iniciais do cultivo. Estas, possivelmente, podem aumentar a concentração de argila em suspensão na água.

Em tanques de aqüicultura semi-intensiva freqüentemente desenvolvem-se densas populações de fitoplâncton (com visibilidade do disco de Secchi $<20 \mathrm{~cm}$ ), em resposta a alta taxa de entrada de nutrientes, que segundo Hargreaves (1998) é vindo do arraçoamento.

Baixas transparências ocorreram no último mês. Infere-se que, foi devido ao incremento alimentar nas porcentagens 5 e 7\% de ração. Boyd e Zimmermann (2000) postularam que alta produtividade natural é geralmente um resultado da alimentação para aumentar a produção de camarão de água doce. Isso causa uma abundância alta de fitoplâncton, baixa concentração de oxigênio dissolvido, estratificação térmica e química, pH alto, concentração excessiva de metabólitos como, amônia, gás carbônico e nitrito.

A absorção de luz foi geralmente acima de $90 \%$. Boyd (1979), estudando viveiros fertilizados, encontrou valores pouco menores.

Apesar de haver estratificação térmica, em algumas coletas, não foi essa a tendência geral durante o cultivo. Boyd (1979) afirma que em sistemas rasos (aproximadamente $1 \mathrm{~m}$ ) e com comprimento de até 0,04ha, os sistemas não apresentam estratificação. Os viveiros, deste estudo, tinham aquela profundidade, como também eram até menores que 0,01 ha. O tempo de residência da água foi cerca de sete dias, possivelmente contribuindo para a não-estratificação. Por outro lado, Kubitza (2003), cita que as correntes de água promovidas pelo fluxo da água que entra no viveiro, promovem um equilíbrio da temperatura.

Malecha (1983) encontraram valores de oxigênio dissolvido na superfície entre 9,52-11,50 e 5,07-8,35 no fundo, adequada para o crescimento de adultos. A ausência de estratificação do oxigênio dissolvido ocorre 
em razão da reduzida profundidade e da ação diária dos ventos (Valenti, 1998), possivelmente não apresentam formação de camada anóxica da coluna da água (EnrichPrast et al., 2004).

A oxigenação e a renovação de água, por fluxo contínuo, são condições importantes ao sucesso dos cultivos semi-intensivos. Uma vez que, evitam o acúmulo de detritos tóxicos e problemas de eutrofização, aumentando a capacidade de suporte do sistema (Boyd e Zimmermann, 2000).

Enfim, a transparência esteve na faixa ideal para o cultivo de Macrobrachium amazonicum, baixas nos últimos meses de cultivo, pois os cultivos interferiram nas mesmas. Quanto à estratificação não houve para a temperatura, o oxigênio e $\mathrm{pH}$ nos viveiros. Portanto, as diferentes taxas alimentares adotadas foram ideais para o cultivo, no que tange à qualidade de água.

Em geral, os tratamentos não determinaram um padrão de variação na produtividade. Os valores mínimos e máximos estiveram entre 500,7 a 793 Kg.ha ${ }^{-1}$ obtidos neste cultivo e podem ser comparados favoravelmente aos dos cultivos descritos por Coelho et al., (1982) em Pernambuco, e Moraes-Valenti e Valenti (2007) em São Paulo.

A produtividade de camarões ocorre em função da quantidade e qualidade do alimento disponível, geralmente abundante e suficiente. Este interfere na transparência. Em estudos realizados com Macrobrachium rosenbergii foi verificado que este depende, fundamentalmente, do alimento natural presente nos viveiros (Valenti e New, 2002). O alimento natural pode ser percebido pela produtividade primária mensurável pela transparência. Não houve relação da produtividade com a transparência comparando-se a despesca total e mista. Por outro lado, observou-se diferenças significativas entre os tratamentos 3 e 7\%, que possivelmente com a adição maior de ração reduz a visibilidade do disco de Secchi.

\section{Agradecimentos}

Ao Centro de Aqüicultura da UNESP pela infraestrutura oferecida para a realização deste trabalho.

\section{Referências}

Boyd, C. E. 1979. Water quality in warmwater fish ponds. Auburn University, Alabama, USA, 359pp.

Boyd, C. E. 1982. Water quality in warmwater fish ponds. Auburn University, Alabama, USA, 359pp.

Boyd, C. E.; Tucker, C. S. 1992. Water quality and pond soil analyses for aquaculture. Auburn University, Alabama, USA, $183 \mathrm{pp}$.

Boyd, C. E.; Zimmermann, S. 2000. Grow-out systems: water quality and soil management. In: New, M. B. \& Valenti, W. C. (Eds). Freshwater prawn culture: The farming of Macrobrachium rosenbergii. Blackwell Science, Oxford, UK, p.221-434.

Coelho, P. A.; Ramos-Porto, M.; Barreto, A. V.; Costa, V. E. 1982. Crescimento em viveiro do camarão-canela (Macrobrachium amazonicum) (Decapoda, Palaemonidae). Revista Brasileira de Zoologia, 1 (1): 45-49.

Correia, E. S.; Pereira, J. A.; Silva, A. P.; Horowitz, A.; Horowitz, S. 2003. Growout of freshwater prawn Macrobrachium rosenbergii in fertilized ponds with reduced levels of formulated feed. Journal of the World Aquaculture Society, 34 (2): 184-191.

Delincé, G. 1992. The ecology of the fish pond ecosystem: With special reference to Africa. Kluwer Academic Publishers, Dordrecht, Netherlands, 230pp.

Enrich-Prast, A.; Bozelli, R. L.; Esteves, F. A.; Meirelles, F. P. 2004. Lagoas costeiras da restinga de Jurubatiba: Descrição de suas variáveis limnológicas. In: Rocha, C. F. D.; Esteves, F. A. \& Scarano, F. R. (Eds). Pesquisas de longa duração na restinga de Jurubatiba Ecologia, História Natural e Conservação. Rima, São Carlos, Brasil, p.245-253.

Esteves, F. A. 1998. Fundamentos de Limnologia. $2^{\text {a }}$ ed. Interciência, Rio de Janeiro, Brasil, 602pp.

Hargreaves, J. A. 1998. Nitrogen biogeochemistry of aquaculture ponds. Aquaculture, 166: 181-212.

Hutchinson, G. E. 1957. A treatise on limnology. v. I. Wiley, New York, USA, 1015pp.

Keppeler, E. C.; Valenti, W. C. 2006. Effects of selective harvest of the amazon river prawn, Macrobrachium amazonicum, on pond water, sediment and effluent. Acta Limnologica Brasiliensia, 18 (2): 110-119.

Kubitza, F. 2003. Qualidade da água no cultivo de peixes e camarões. ESALQ/USP, Jundiaí, Brasil, 229pp.

Margalef, R. 1983. Limnología. Omega S.A., Barcelona, España, 1010pp.

Malecha, S. R. 1983. Commercial pond production of the freshwater prawn, Macrobrachium rosenbergii, in Hawaii. In: Mcvey, J. P. \& Moore J. R. (Eds). CRC Handbook of Mariculture, VI Crustacean Aquaculture. CRC Press, Florida, USA, 442pp.

Moraes-Valenti, P. M. C.; Valenti, W. C. 2007. Effect of intensification on grow-out of the Amazon River Prawn, Macrobrachium amazonicum. Journal of the World Aquaculture Society, 38: 516-526.

Piedrahita, R. H.; Brune, D. E.; Tchobanoglous, G.; Orlob, G. T. 1984. A general model of the aquaculture pond ecosystem. Journal of the World Mariculture Society, 15: 355-366. 
Poole, H. H.; Atkins, W. R. G. 1929. Photo-electric measurements of submarine illumination throughout the year. Journal of the Marine Biological Association of the United Kingdom, 16 (1): $297-$ 324.

Ribeiro, R. P. 2001. Construção de tanques. In: Moreira, H. L. M.; Vargas, L.; Ribeiro, R. P. \& Zimmermann, S. (Eds). Fundamentos da moderna aquicultura. Ulbra, Canoas, Brasil, p.45-52.

SAS. 2001. SAS/STAT User's Guide: Statistics. Version 8.2. SAS Institute Inc, Cary, NC.

Souza, V. L.; Sipaúba-Tavares, L. H.; Urbinati, E. C. 2001. Manejo alimentar e tempo de residência da água em viveiros de pacu (Piaractus mesopotamicus). Ciência Animal Brasileira, 1 (2): 115-121.

Statistica. 1996. Statsoft. Versão 6.0.

Valenti, W. C. 1985. Cultivo de camarões de água doce. Nobel, São Paulo, Brasil, 82pp.

Valenti, W. C. 1998. Sistemas de produção na fase de crescimento final. In: Valenti, W. C. (Ed.). Carcinicultura de água doce. Tecnologia para a produção de camarões. IBAMA, Brasília, Brasil, p.165-177.
Valenti, W. C.; New, M. B. 2000. Grow-out systems-monoculture. In: New, M. B. \& Valenti, W. C. (eds). Freshwater prawn culture: the farming of Macrobrachium rosenbergii. Blackwell Science, Oxford, UK, p.157-176.

Weidenbach, R. P. 1980. Dietary components of prawns reared in Hawaiian ponds. Proceedings of the Giant Prawn Conference, Bangkok, Thailand, p.149-158.

Wetzel, R. G. 1981. Limnología. Ômega S.A, Barcelona, España, 679pp.

Wetzel, R. G.; Likens, G. E. 1991. Limnological analysis. Springer-Verlag, New York, USA, 391pp.

Zar, J. H. 1984. Biostatistical Analysis. $2^{\text {nd }}$ ed. Prentice Hall, Englewood Cliffs, New Jersey, USA, 718pp.

Zimmermann, S. 1998. Manejo da fase de crescimento final. In: Valenti, W. C. (ed.). Carcinicultura de água doce. Tecnologia para a produção de camarões. IBAMA, Brasília, Brasil, p.191215. 